\title{
Technically simplified peroral endoscopic myotomy: blunt dissection making submucosal tunneling fast and safe
}

As a primary treatment for patients with achalasia [1], peroral endoscopic myotomy (POEM) is a technically demanding procedure [2]. To perform a high-quality POEM and to prevent complications such as leakages, infections, major bleeding, and gas-related events, the establishment of a submucosal tunnel and the integrity of the mucosal flap are crucial [3, 4]. We present a blunt dissection technique to achieve rapid and safe submucosal tunneling in the aim to build a technically simplified POEM, which we call blunt POEM ( Video 1 ).

A 58-year-old woman suffered from solid food dysphagia and regurgitation. The endoscopy presented two surgical scars ( $\triangleright$ Fig. 1 a,b) and a contraction ring at the lower esophagus ( $\mathbf{F i g} \mathbf{1} \mathbf{c}$ ). The patient was diagnosed with type II achalasia under barium esophagography ( $\triangleright$ Fig. 1 d) and high resolution esophageal manometry. We decided to perform POEM on the patient. Briefly, a mucosal incision was made on the posterior esophageal wall ( $\triangleright$ Fig.2a). Once the endoscope was maneuvered into the submucosal space, the transparent cap was applied to bluntly dissect the fibers and thus establish a submucosal tunnel ( $\triangleright$ Fig. 2 b). The blunt dissection was so efficient that it took only 2 minutes to establish a 10 -cm tunnel ( $>$ Fig. 2 c). After the submucosal tunnel was extended 2 $\mathrm{cm}$ into the proximal stomach, the selective inner circular myotomy and fullthickness myotomy were conducted ( $\triangleright$ Fig.2d). Finally, the mucosal entry was closed using endoclips (> Fig.2e). After the procedure, the lower esophageal sphincter (LES) was open and the endoscope smoothly passed the cardia ( Fig. 2f).

The simplest strategy to establish a tunnel is to dissect the submucosal fibers without a knife, and the most effective way to prevent complications is to preserve the intact mucosal flap [5]. In the current method of blunt POEM, we devel-

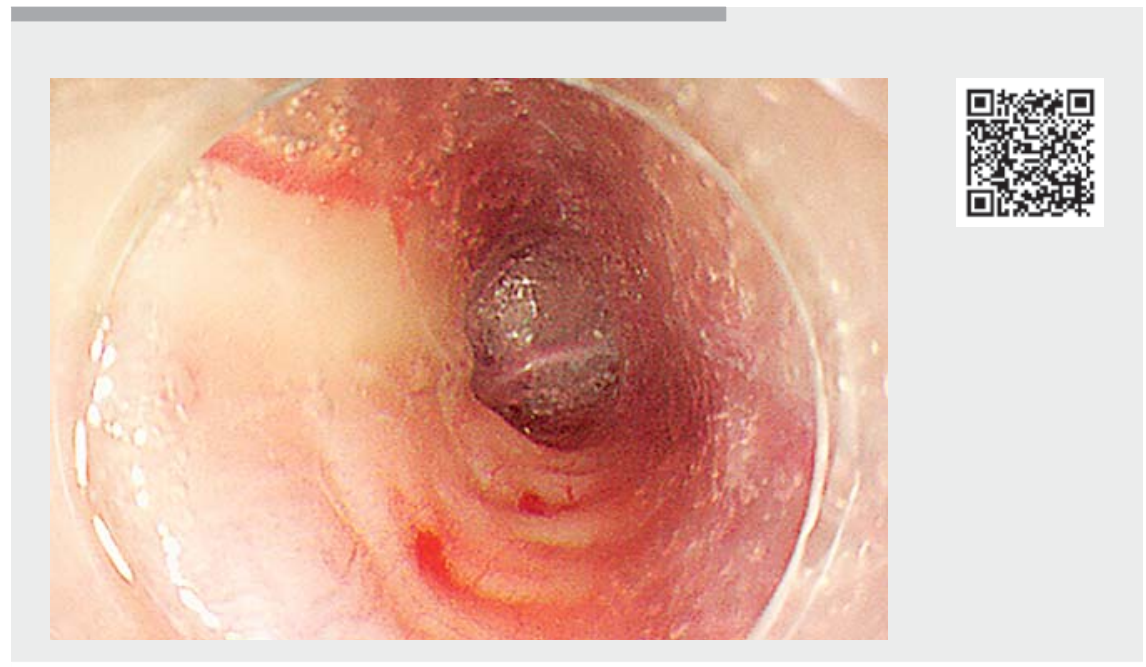

Video 1 The establishment of a novel peroral endoscopic myotomy (POEM) using a blunt dissection-based submucosal tunneling technique.

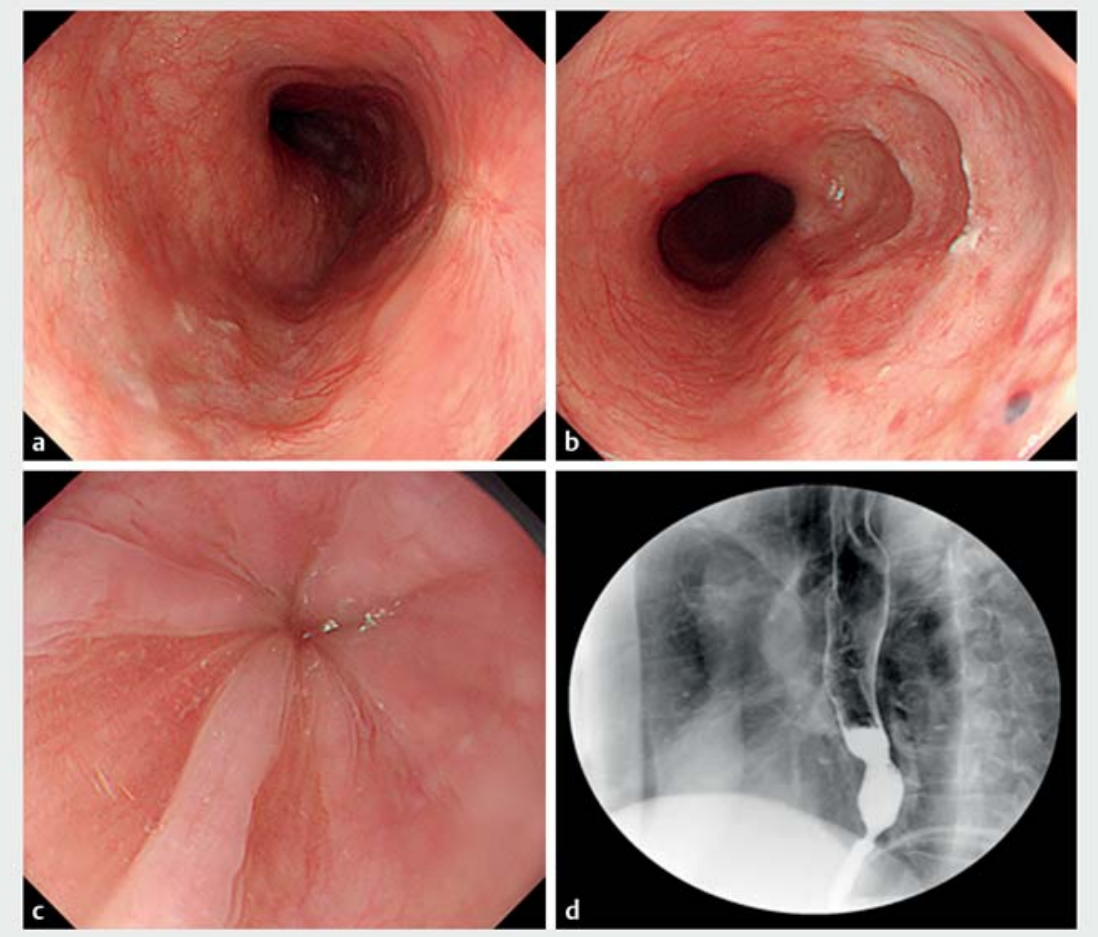

- Fig. 1 The diagnosis of achalasia after endoscopic submucosal dissection of early esophageal cancer. a One surgical scar on the right esophageal wall $24 \mathrm{~cm}$ away from the incisors. b Another esophageal scar $34 \mathrm{~cm}$ away from the incisors. c Contractile closed cavity was seen at the lower esophagus under endoscopy. $\mathbf{d}$ A beaklike narrow ring presented at the lower esophagus on barium esophagography. 


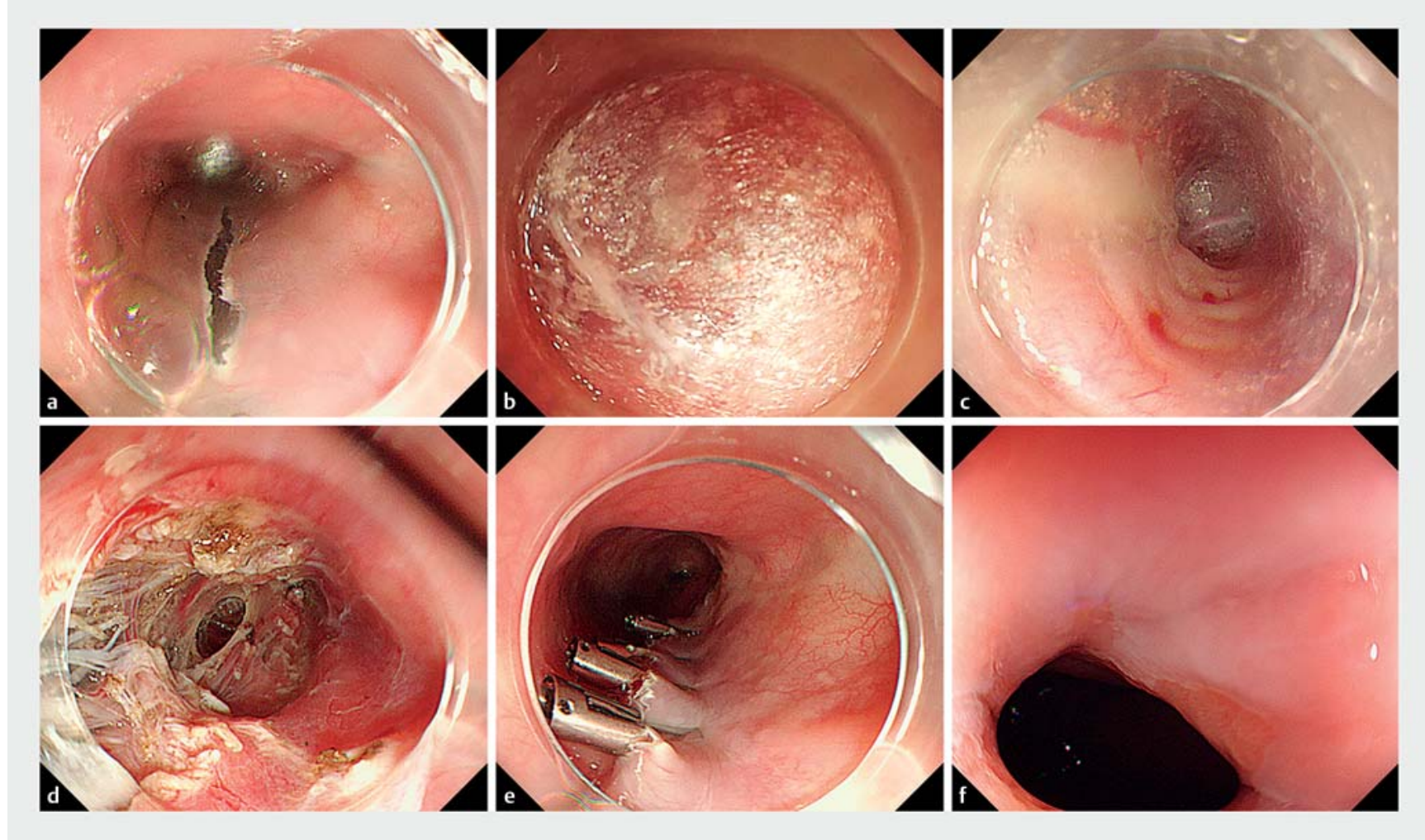

- Fig. 2 The establishment of a novel peroral endoscopic myotomy (POEM) using blunt dissection-based submucosal tunneling technique. a A $1.5-\mathrm{cm}$ to $2-\mathrm{cm}$ longitudinal mucosal opening was made after a submucosal cushion was created with the methylene blue saline solution. b The endoscope with a transparent cap fitted on its tip was drilled into the submucosal layer to create the working tunnel by bluntly dissecting fibers with the transparent cap.c A wide submucosal tunnel was established rapidly and safely as care was taken with the orientation of the endoscope. $\mathbf{d}$ Myotomy was performed from $2 \mathrm{~cm}$ below the mucosal opening to the end of the submucosal tunnel. e The mucosal opening was closed using endoscopic clips. f After the procedure, the cardia was relaxed and the endoscope was passed through it smoothly.

oped blunt dissection to simultaneously create a safe tunnel and preserve the mucosal flap. Compared with traditional POEM, blunt POEM is more easily maneuverable and less time-consuming for the treatment of achalasia.

Endoscopy_UCTN_Code_TTT_1AO_2AN

\section{Competing interests}

The authors declare that they have no conflict of interest.

The authors

Xiao Hu*, Ying-hui Zhang*, Xiao-gang Liu, Sheng-xi Han, Wei-hui Liu ${ }^{\circ}$

Department of Gastroenterology and Hepatology, Sichuan Academy of Medical Sciences \& Sichuan Provincial People's Hospital, School of Medicine, University of Electronic

\footnotetext{
* Contributed equally to this work.
}

Science and Technology of China, Chengdu, Sichuan Province, China

\section{Corresponding author}

\section{Wei-hui Liu, MD}

Department of Gastroenterology and Hepatology, Sichuan Academy of Medical Sciences \& Sichuan Provincial People's Hospital, School of Medicine, University of Electronic Science and Technology of China, Chengdu, Sichuan Province, 610072, China audiliu12@163.com

\section{References}

[1] Ponds FA, Fockens P, Lei A et al. Effect of peroral endoscopic myotomy vs. pneumatic dilation on symptom severity and treatment outcomes among treatment-naive patients with achalasia: a randomized clinical trial. JAMA 2019; 322: 134-144

[2] Khashab MA, Sethi A, Rosch T et al. How to perform a high-quality peroral endoscopic myotomy? Gastroenterology 2019; 157: 1184-1189

[3] Familiari P, Landi R, Mangiola F et al. Endoscopic treatment of intramural fistula and mucosal tear after peroral endoscopic myotomy. Endoscopy 2021; 53: E25-E26

[4] Nabi Z, Ramchandani M, Chavan R et al. Peroral endoscopic myotomy in treatmentnaive achalasia patients versus prior treatment failure cases. Endoscopy 2018; 50: 358-370

[5] Yamasaki M, Kume K, Yoshikawa I et al. A novel method of endoscopic submucosal dissection with blunt abrasion by submucosal injection of sodium carboxymethylcellulose: an animal preliminary study. Gastrointest Endosc 2006; 64: 958-965

\section{Bibliography}

Endoscopy 2022; 54: 724-725

DOI 10.1055/a-1532-1759

ISSN 0013-726X

published online 9.7.2021

(c) 2021. Thieme. All rights reserved.

Georg Thieme Verlag KG, Rüdigerstraße 14, 70469 Stuttgart, Germany 